\title{
EVOLUÇÃO DA RESISTÊNCIA IN VITRO DO PLASMODIUM FALCIPARUM A ANTIMALÁRICOS EM ÁREA DE PROSPECÇÃO DE OURO NO ESTADO DO AMAPÁ, ENTRE
} 1983 E 1990.

\begin{abstract}
A.A. Couto, V.S. Calvosa, J.E. Lima e J.M. Souza
Este estudo avalia a evolução da sensibilidade in vitro do Plasmodium falciparum em uma área de prospecção de ouro no Estado do Amapá no período de 1983 a 1990. Foram efetuados 75 testes para cloroquina e quinino, 74 para amodiaquina e 76 para mefloquina. Os resultados revelaram $81 \%$ de resistência à cloroquina e $27 \%$ para a amodiaquina, enquanto que para quinino e mefloquina não foram evidenciadas cepas resistentes. Contudo, para estas duas últimas drogas identificou-se uma crescente perda da sensibilidade ao longo do tempo. Aparentemente observa-se uma associação entre resistência à cloroquina e a diminuição da sensibilidade ao quinino.
\end{abstract}

Palavras-chaves: Plasmodium falciparum. Resistência in vitro. Cloroquina. Amodiaquina.

A resistência do Plasmodium falciparum às drogas antimaláricas representa um dos maiores obstáculos para o êxito de combate e controle da malária em muitas áreas onde a doença ocorre.

No Brasil, já em 1910, registrava-se o primeiro insucesso no tratamento da malária com o quinino ${ }^{13}$.

Para a cloroquina o primeiro registro de resistência na América do Sul ocorreu no final dos anos 50, na Colômbia ${ }^{28}$ eno Brasil ${ }^{1821}$. Atualmente a resistência às drogas é um fenômeno muito comum em diversas áreas da região amazônica brasileira, não só para as 4-aminoquinoleínas como para quinolinosmetanóis ${ }^{1} 691619$. Além disso, mais recentemente no Brasil, especialmente na região amazônica brasileira, tem sido demonstrada a ocorrência de cepas multi-resistentes ${ }^{4}$. No caso específico da Amazônia Brasileira cepas de $P$. falciparum isoladas em várias áreas mostraram $83 \%$ de resistência à cloroquina, $56 \%$ e $51 \%$ ao quinino e amodiaquina, respectivamente e $2,3 \%$ de resistência à mefloquina ${ }^{19}$. No Estado do Acre, em área de colonização, essa resistência à cloroquina

\footnotetext{
Programa de Malária, Instituto Evandro Chagas, Fundação Nacional de Saúde (FNS), Belém, PA.

Apoio financeiro da UNDP/WORLD/BANK/WHOSPECIAL PROGRAMME-TDR,PROJETO(870284).

Enderę̧o para correspondência: Dr. Alvaro Couto. Av. Almirante Barroso 492, Marco, 66090-000 Belém, PA.

Recebido para publicação em $03 / 08 / 93$.
}

e amodiaquina foi $84 \%$ e $73 \%$, respectivamente e para o quinino foi de $2,3 \%$; para mefloquina todos os testes indicaram sensibilidade ${ }^{9}$.

A migração na Amazônia tem sido considerada como fator determinante do aumento e disseminação da malária na região ${ }^{10}$, sobretudo a movimentação intra e extra-amazônica, particularmente relacionada aos núcleos de colonização e áreas de mineração.

O presente estudo avalia a evolução da resposta in vitro do $P$. falciparum para quatro drogas de uso comum no tratamento da malária em uma área do Estado do Amapá - Brasil, no período de 1983 a 1990.

\section{MATERIAL E MÉTODOS}

Área de estudo: O estudo foi desnvolvido no garimpo do Lourenço, município de Calçoene, Estado do Amapá. Essa região caracteriza-se pela intensa e exclusiva atividade de prospeç̧ão de ouro a céu aberto com uma população instável, variando entre 6.000 a 10.000 habitantes, vivendo de forma precária. Devido as condições habitacionais o contato vetor homem é muito facilitado, o que pode ser um dos fatores epidemiológicos mais importantes eo principal responsável pelo alto nível de transmissão da malária nesta região. Segundo Sawyer $^{20}$, a região se enquadra na classificação "Área rural extrativista garimpo aberto - tipo2". 
Couto AA, Calvosa VS, Lima JE, Souza JM. Evolução da resistência in vitro do Plasmodium falciparum a antimaláricos em área de prospeç̧ão de ouro no Estado do Amapá, entre 1983 e 1990. Revista da Sociedade Brasileira de Medicina Tropical 26:215-220, out-dez, 1993.

Amostras: As amostras (cepas) do parasito foram isoladas de 76 indivíduos apresentando idades entre 13 e 48 anos, de ambos os sexos, porém com absoluta predominância deadultos do sexo masculino correspondendo a $92 \%$ e $88 \%$, respectivamente, o que caracteriza o perfil das populações de áreas de garimpagem de ouro na região amazônica brasileira. Os participantes declararam o não uso de drogas antimaláricas nas últimas quatro semanas, além de darem consentimento oral para sua inclusão no estudo. Todos os indivíduos eram portadores exclusivamente de $P$. falciparum, com parasitemia superior a 500 parasitos por milímetro cúbico de sangue, e, não apresentando quadro de malária grave. Quando a parasitemia era igual ou superior a $1 \%$ a amostra de sangue era diluída ajustando-se para o intervalo de 0,5 a $0,7 \%$, conforme metodologia preconizada.

De cada indivíduo foram obtidos $10 \mathrm{ml}$ de sangue, por punção venosa, utilizando-se ampolas de VACUTAINER (Becton Dickinson) heparinizadas. Com este material realizaram-se, além dos microtestes, o isolamento de cepas para cultivo in vitro e criopreservação.

Microtestes: Foram realizados com base no método descrito por Rieckmann e cols ${ }^{17}$. Na primeira fase do estudo (1983 a 1986) as drogas foram diluídas no momento da realização dos testes, partindo-se de uma solução "estoque" fornecida pela Organização Mundial de Saúde (OMS), como descrito em estudos anteriores do nosso grupo sobre a resistência in vitro do $P$. falciparum em várias localidades da região Amazônica ${ }^{19}$. Utilizaram-se as seguintes concentrações para cada droga: cloroquina (difosfato) 1,0 a $32,0 \times 10-8 \mathrm{M}$; mefloquina 0,5 a $16,0 \times 10-8 \mathrm{M}$; amodiaquina 0,25 a $16,0 \times 10-8 \mathrm{M}$ e quinino (sulfato) 3,9 a $250,0 \times 10-$ 8M. Já na segunda fase, de 1987 a 1990, os testes foram processados em placas pré-doseadas com as diferentes drogas também fornecidas pela OMS. Foram usadas as seguintes concentrações para cada uma das drogas: cloroquina 1,0 a $64,0 \mathrm{pmol}$; amodiaquina 0,25 a $16,0 \mathrm{pmol}$; quinino 4,0 a 256,0 pmol e mefloquina 2,0 a 128,0 pmol por orifício da placa.

Após a coleta, em ambas as fases, a amostra sanguínea foi lavada três vezes em meio de cultivo RPMI 1640 (Gibco) incompleto. Durante a primeira fase uma alíquota do sangue infectado foi incorporada ao meio de cultivo o qual foi preparado acrescido das várias concentrações das drogas, enquanto que na segunda fase a suspensão de hemácias parasitadas foi aplicada diretamente nos orifícios de cada placa pré-doseada frente as diversas concentrações dessas drogas. Em seguida as placas foram incubadas conforme metodologia do cultivo contínuo in vitro ${ }^{23}$ durante 24 a 48 horas. Ao final deste período foram preparadas gotas-espessas de sangue de cada orifício e coradas pelo método de Giemsa. Para cada concentração das drogas testadas contou-se o número de pré-esquizontes e esquizontes encontrados em um total de $\mathbf{2 0 0}$ formas assexuadas comparando-se cada orifício contendo droga com um orifício controle sem presença de droga.

Avaliação da suscetibilidade: Foi realizada com base nas instruções da OMS 14152627 . Foram consideradas como cepas resistentes àquelas que apresentaram maturação de esquizontes em concentrações iguais ou superiores a: cloroquina 8,0pmol; amodiaquina 4,0pmol; quinino 128,0pmol e mefloquina 64,0pmol.

Os resultados foram analisados estatisticamente através do teste qui-quadrado (X2) sendo considerado o nível de significância para $p=0,01$.

\section{RESULTADOS}

Dos 76 testes efetuados para as drogas em estudo, 75 apresentaram boas condições para avaliação para cloroquina e quinino, 74 para amodiaquina e 76 para mefloquina. Observou-se $81 \%$ de resitência à cloroquina, $27 \%$ para amodiaquina, enquanto que para as drogas quinino e mefloquina os testes não revelaram cepas resistentes (Tabela 1). No entanto, entre as amostras estudadas, $10,6 \%$ e $1,3 \%$ respectivamente, para quinino e mefloquina, tiveram seu crescimento inibido na concentração limite da sensibilidade (Tabela 1). 
Couto AA, Calvosa VS, Lima JE, Souza JM. Evolução da resistência in vitro do Plasmodium falciparum a antimaláricos em área de prospecção de ouro no Estado do Amapá, entre 1983 e 1990. Revista da Sociedade Brasileira de Medicina Tropical 26:215-220, out-dez, 1993.

Tabela 1 - Sensibilidade in vitro de isolados de Plasmodium falciparum à cloroquina, amodiaquina, quinino e mefloquina na região do Lourenço-AP no periodo de 1983 à 1990, medida pela completa inibição da maturação (CIM) de esquizontes na presença de diferentes concentrações das drogas.

\begin{tabular}{|c|c|c|c|c|c|c|c|c|}
\hline \multirow{3}{*}{$\begin{array}{l}\text { Concentrações } \\
\text { das drogas } \\
\text { (pmol/orifício) }\end{array}$} & \multicolumn{8}{|c|}{ Isolados testados } \\
\hline & \multicolumn{2}{|c|}{ cloroquina } & \multicolumn{2}{|c|}{ amodiaquina } & \multicolumn{2}{|c|}{ quinino } & \multicolumn{2}{|c|}{ mefloquina } \\
\hline & $n^{0}$ & $\%$ & $n^{0}$ & $\%$ & $n^{0}$ & $\%$ & $n^{0}$ & $\%$ \\
\hline 0,25 & $\mathrm{nr}$ & & 2 & 2,7 & $\mathrm{nr}$ & & $\mathrm{nr}$ & \\
\hline $0 ; 50$ & $\mathrm{nr}$ & & 4 & 5,4 & $\mathrm{nr}$ & & $\mathrm{nr}$ & \\
\hline 1,00 & 0 & 0,0 & 7 & 9,5 & $\mathrm{nr}$ & & $\mathrm{nr}$ & \\
\hline 2,00 & 0 & 0,0 & 16 & 21,6 & $\mathrm{nr}$ & & 48 & 63,2 \\
\hline 4,00 & 4 & 5,3 & 25 & $33,7^{*}$ & 1 & 1,3 & 24 & 31,6 \\
\hline 8,00 & 10 & $13,3^{*}$ & 9 & 12,2 & 1 & 1,3 & 2 & 2,6 \\
\hline 16,00 & 26 & 34,6 & 11 & 14,9 & 12 & 16,0 & 1 & 1,3 \\
\hline 32,00 & 28 & 37,5 & $\mathrm{nr}$ & & 32 & 42,8 & 1 & 1,3 \\
\hline 64,00 & 7 & 9,3 & $\mathrm{nr}$ & & 21 & 28,0 & 0 & $0,0 *$ \\
\hline 128,00 & $\mathrm{nr}$ & & $\mathrm{nr}$ & & 8 & 10,6 & 0 & 0,0 \\
\hline 256,00 & $\mathrm{nr}$ & & $\mathrm{nr}$ & & 0 & 0,0 & $\mathrm{nr}$ & \\
\hline
\end{tabular}

* Limite da sensibilidade; nr Teste não realizado

Quando as cepas resitentes à cloroquina foram ordenadas em níveis crescentes de inibição de 16,0 , 32,0 e 64,0pmol e comparadas quanto a resposta às demais drogas analisadas, observou-se uma associação positiva entre a resistência das cepas à cloroquina e a redução da sua sensibilidade ao quinino ( $\mathrm{P}=0,01)$. Por outro lado, a comparação da resposta entre as drogas cloroquina com amodiaquina e cloroquina com mefloquina não demonstrou uma relação positiva estatisticamente significativa (Tabela 2).

Tabela 2 - Respostas de cepas resistentes à cloroquina a outros antimaláricos expressas em média da concentração mínima inibitória (CMI) capaz de determinar a completa inibição da maturação de esquizontes.

\begin{tabular}{cccc}
\hline \multicolumn{4}{c}{ Drogas } \\
\hline $\begin{array}{c}\text { cloroquina } \\
\text { (pmol) }\end{array}$ & $\begin{array}{c}\text { quinino } \\
\text { (pmol)* }\end{array}$ & $\begin{array}{c}\text { amodiaquina } \\
\text { (pmol)* }\end{array}$ & $\begin{array}{c}\text { mefloquina } \\
(\mathrm{pmol})^{*}\end{array}$ \\
\hline 16,0 & 38,8 & 4,6 & 2,7 \\
32,0 & 65,2 & 6,5 & 3,6 \\
64,0 & 58,6 & 8,6 & 7,3 \\
\hline
\end{tabular}

* Média das CMI's

cloroquina $x$ quinino $\quad(x 2=12,15)$

cloroquina $x$ amodiaquina $(x 2=1,54)$

cloroquina $x$ mefloquina $(x 2=0,3)$

Qui-quadrado para $\mathrm{gl}=2 ; \mathrm{P}=0,01$
O estudo da evolução da resistência in vitro do $P$. falciparum em cepas isoladas na região de Lourenço, ao longo do período estudado, evidenciou uma relativa estabilidade da resposta dos parasitos à cloroquina e ao quinino. Entretanto, a prevalência de resistência à cloroquina é evidente em todos os anos, exceto em 1986. Já para o quinino constatase uma gradual perda de sensibilidade (Figura 1).

Para a amodiaquina destacam-se dois períodos onde houve prevalência de cepas resistentes, notadamente em 1983/85 e 1988/90, enquanto que no biênio $1986 / 87$ as cepas apresentaram $100 \%$ de sensibilidade. A sensibilidade à mefloquina foi total ao longo de todo o período de observação. Contudo, evidenciou-se uma aparente perda de sensibilidade a essa droga no período de 1988/90. Identificaram-se em 1990 cepas apresentando inibição de maturação na concentração limite da sensibilidade, ou seja, 32,0pmol, o que sugere a possibilidade da emergência de cepas resistentes à mefloquina na região do Lourenço no Estado do Amapá.

\section{DISCUSSÃO}

A intensidade da garimpagem de ouro no Estado do Amapá, particularmente na região do Lourenço, tem evoluído de tal forma que hoje é muito significativo o número de pessoas que vivem direta 
Couto AA, Calvosa VS, Lima JE, Souza JM. Evolução da resistência in vitro do Plasmodium falciparum a antimaláricos em área de prospecção de ouro no Estado do Amapá, entre 1983 e 1990. Revista da Sociedade Brasileira de Medicina Tropical 26:215-220, out-dez, 1993.

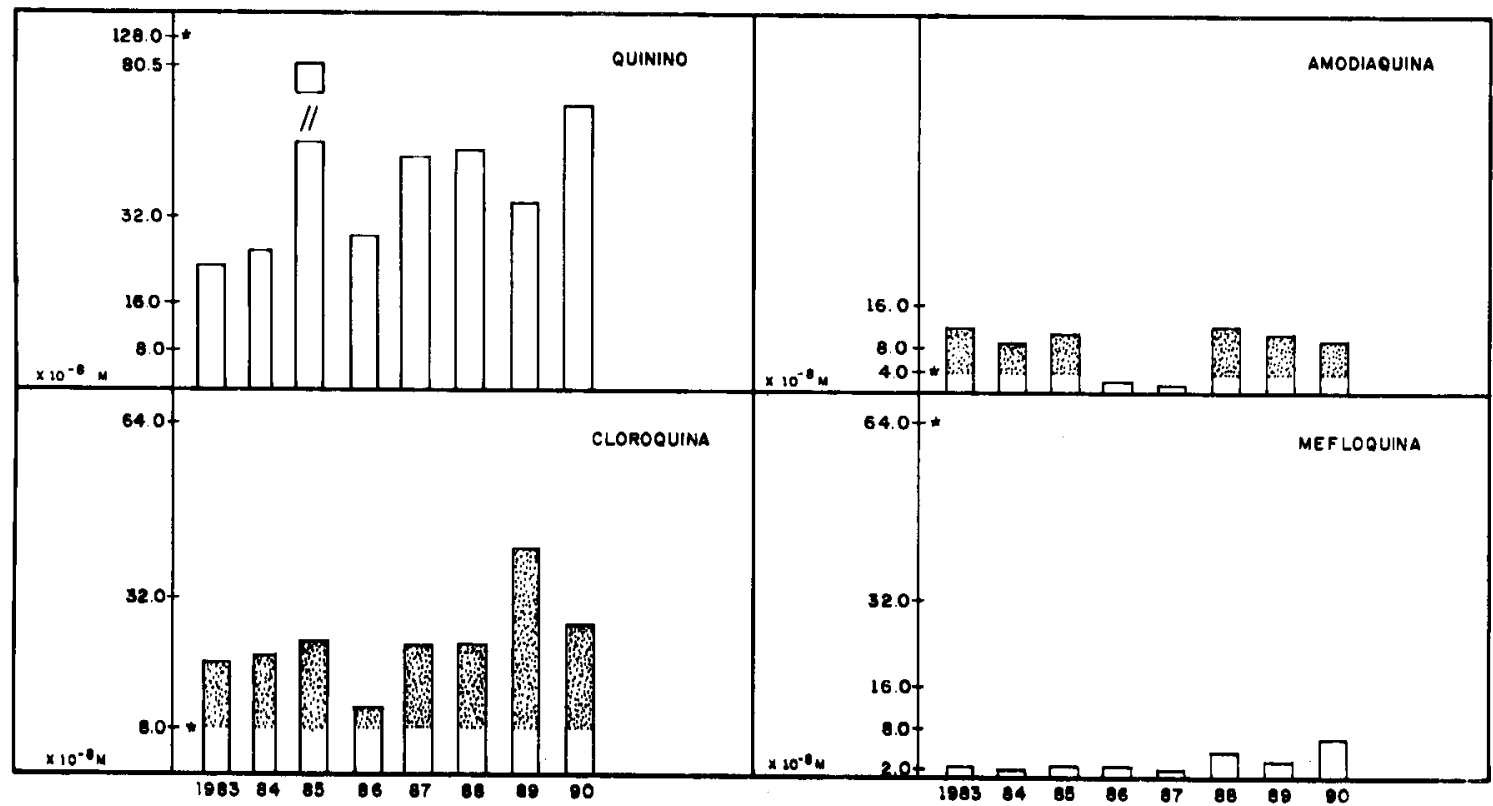

- Limlar do censibilidode

Resletente

Sensivel

Figura 1-Evoluçao da resistência in vitro de cepas de P. falciparum a diferentes antimaláricos na região de Lourenço, AP de 1983 a 1990.

ou indiretamente dessa atividade. É freqüente a presença de trabalhadores antigos com experiência de vários episódios de malária que apresentam quadro de infeç̧ão subclínica. Os recém-chegados em geral apresentam alta suscetibilidade e elevadas parasitemias sendo que algumas vezes desenvolvem malária grave. Provavelmente, como conseqüência da intensa migração, a incidência da malária no Estado do Amapá elevou-se de 2.812 casos em 1981 para 11.156 em 1989, o que coincide com a intensificação da exploração de ouro na região de Lourenço ${ }^{11} 12$.

Vários fatores estão associados à emergência e à disseminação da resistência às drogas utilizadas para o tratamento da doença mas provavelmente o mais importante entre eles é a pressão de drogas. Muitos e diferentes antimaláricos são geralmente utilizados nos garimpos em dosagem subcurativas. A possível vantagem biológica dos parasitos resistentes sobre os sensíveis, além de outros fatores ligados ao hospedeiro como a resposta imunológica que é aparentemente essencial a uma terapêutica eficaz ${ }^{22}$, podem contribuir com o aumento da resistência.
A automedicação é muito freqüente na região do Lourenço, inclusive a utilização da mefloquina detectada desde 1986 e mais recentemente os derivados da artemisinina, conforme nossas observações realizadas no local. Possivelmente estes sejam os principais responsáveis pela perda da eficácia destas drogas, inclusive a mefloquina, detectada nos últimos anos relativos ao presente estudo.

Nossos resultados mostram a evolução da resistência na região do Lourenço. Aí evidenciouse um elevado grau de resitência in vitro à cloroquina (81\%) similar àquela ja descrita por nosso grupo em estudos anteriores em outras áreas da Amazônia Oriental ${ }^{4}{ }^{1924} \mathrm{eOcidental}^{6}$, bem como, nesta última, por outros autores ${ }^{1916}$. Esta similaridade sugere que a resitência à cloroquina, na região amazônica brasileira, tem ampla dispersão e está uni formemont distribuída nas áreas de elevada transmissão $\therefore$ malária.

A resistência à amodiaquina foi da ordem de $27 \%$ das cepas analisadas neste estudo, indicando que esta 'roga apesar de ser uma 4-aminoquinoleína parece mais eficaz que a cloroquina. Estudos in 
Couto AA, Calvosa VS, Lima JE, Souza JM. Evolução da resistência in vitro do Plasmodium falciparum a antimaláricos em área de prospecção de ouro no Estado do Amapá, entre 1983 e 1990. Revista da Sociedade Brasileira de Medicina Tropical 26:215-220, out-dez, 1993.

vitro realizados por nosso grupo demonstram expressiva eficácia da amodiaquina, no tratamento de malária falciparum, principalmente quando associada à tetraciclina. Tal associação induziu $100 \%$ de resposta S/RI em 18 pacientes oriundos de áreas endêmicas da Amazônia Brasileira, inclusive da região do Lourenço-AP, onde são freqüentes cepas cloroquino-resistentes ${ }^{3}$.

Não se registraram cepas resistentes ao quinino sugerindo ser esta uma droga bastante efetiva no tratamento da malária causada pelo $P$. falciparum, apesar dos seus marcantes efeitos colaterais freqüentemente presentes ${ }^{25}$. Também não foi constatada resistência à mefloquina, havendo no entanto redução da sensibilidade em $1,3 \%$ das amostras analisadas, que foram inibidas em presença da concentração limite da sensibilidade. Este fato sugere uma tendência ao aparecimento da resistência a esta droga, fato este já relatado, in vitro e in vivo apenas em cepas isoladas em áreas ja Amazônia Ocidental $^{25}$. A perda acentuada da sensibilidade à mefloquina detectada em cepas isoladas no Lourenço sugere uma tendência ao surgimento de resistência nesta área.

Knowles e cols ${ }^{8}$, classificaram os parasitos conforme a resposta para cada droga e demonstraram que cepas resistentes à cloroquina possuem reduzida sua sensibilidade ao quinino. Não observaram, contudo, correlação com a atividade daquela e a da mefloquina. Nossos resultados mostram também associação entre cepas resistentes à cloroquina e a diminuição da sensibilidade ao quinino $(P=0,01)$, mas, ausência de uma relação com a perda da sensibilidade à amodiaquina, que também é uma 4aminoquinoleína, bem como à mefloquina.

Considerando esse fenômeno em que a perda da sensibilidade ao quinino está relacionada com o aumento da resistência à cloroquina, é possível que uma população de parasitos que exiba este perfil esteja, eventualmente, colocando em risco a eficácia da mefloquina, uma vez que esta é estruturalmente semelhante ao quinino?

Maiores estudos são necessários para efetivamente se elucidar o mecanismo pelo qual emergem cepas de $P$. falciparum expressando esta característica de resitência cruzada para cloroquina e quinino e determinar o grau de risco de comprometer drogas antimaláricas como a mefloquina.

\section{SUMMARY}

This study evaluates the sensitivity of $\mathrm{P}$. falciparum in vitro to antimalarial drugs in an area of gold mining exploration in Amapa State during the period of 1983 to 1990. The following tests were done, 75 in vitro studies with chloroquine and quinine, 74 with amodiaquine and 76 with mefloquine. The results showed $81 \%$ of resistance to chloroquine and $27 \%$ to amodiaquine while resistant strains to quinine and mefloquine were found. Also for these two quinolinomethanols a loss of sensitivity was noticed in the period of study. An association between resistance to chloroquine and decrease of sensitivity to quinine was also evident with the same strains.

Key-words: Malaria. Plasmodium falciparum. In vitro resistance. Gold prospectors.

\section{AGRADECIMENTOS}

Os autores agradecem o apoio recebido da FNS - Coordenadoria Regional do Amapá e a Mineração Novo Astro S/A, sem o que não seria possível a realização deste estudo. Agradecem também à Dra. Luiza Moutinho do Centro de Hemoterapia do Pará - HEMO-PA pela constante colaboração e aos senhores Edvaldo Santa Rosa e José Maria Nascimento pelo auxílio nas atividades de campo. Especial agradecimento à Dra. Antoniana Krettli pela revisão crítica do manuscrito.

\section{REFERENCIAS BIBLIOGRÁFICAS}

1. Alecrim WD, Robles CRQ, Reys S, Nascimento JAP. Testes in vivo da resitência do $P$. falciparum a cloroquina, amodiaquina e sulfadoxina + pirimetamina na Amazônia. In: Resumos do XXII Congresso da Sociedade Brasileira de Medicina Tropical, Belo Horizonte p.90, 1986.

2. Boulos M, Segurado AAC, Dutra AP, Shiroma M, Ferreira I. Resistência do P. falciparum à associação mefloquina + sulfadoxina + pirimetamina. $I n$ : Resumos do XXII Congresso da Sociedade Brasileira de Medicina Tropical, Belo Horizonte p.88, 1986.

3. Calvosa VS, Couto AA, Souza JM. Associação amodiaquina + tetraciclina no tratamento da malária falciparum em pacientes com malária aguda oriundos de áreas com comprovada resistência à cloroquina. 
Couto AA, Calvosa VS, Lima JE, Souza JM. Evolução da resistência in vitro do Plasmodium falciparum a antimaláricos em área de prospeç̧ão de ouro no Estado do Amapá, entre 1983 e 1990. Revista da Sociedade Brasileira de Medicina Tropical 26:215-220, out-dez, 1993.

In: Resumos do XXVIII Congresso da Sociedade Brasileira de Medicina Tropical, Belém p. 14, 1992.

4. Couto AA, Calvosa VS, Santos MA, Souza JM. Resistência in vitro de cepas de Plasmodium falciparum isoladas no Sul do Estado do Pará, em diferentes períodos: emergência de casos de multiresistência. Revista da Sociedade Brasileira de Medicina Tropical 26:5-9, 1993.

5. Di Santi SM, Boulos M, Neves VLFC, Dutra AP, Barata LCB. Aparecimento e proliferação de resistência de $P$. falciparum à mefloquina. In: Resumos do XXII Congresso da Sociedade Brasileira de Medicina Tropical, Belo Horizonte p.89, 1986.

6. Di Santi SM, Boulos M, Vasconcelos MA, Oliveira SG, Couto AA, Rosário VE. Caracterização de cepas de Plasmodium falciparum do Estado de Rondônia, Brasil, utilizando microtestes de sensibilidade aos antimaláricos, tipificação enzimática e anticorpos monoclonais. Revista do Instituto de Medicina Tropical de São Paulo 29:142147, 1987.

7. Howells RE. Advances in chemotherapy. British Medical Bulletin 38:193-199, 1992.

8. Knowles G, Davisson WL, Jolley D, Alpers MP. The relationship between the in vitro response of Plasmodium falciparum to chloroquine, quinine and mefloquine. Transaction of the Royal Society of Tropical Medicine and Hygiene 78:146-150, 1984.

9. Kremsner PG, Zotter GM, Feldmeir H, Graninger W, Kollaristsch M, Wiedermann G, Rocha RM, Wernsdorfer WH. In vitro drug sensivity of Plasmodium falciparum in Acre, Brazil. Bulletin of the World Health Organization 67:289-293, 1989.

10. Marques AC. Migration and the dissemination of malaria in Brazil. Memórias do Instituto Oswaldo Cruz 81(Supl 2):17-30, 1986.

11. Ministério da Saúde/Superintendência de Campanhas de Saúde Pública-SUCAM-Diretoria Regional do Amapá. Dados epidemiológicos da malária no Estado do Amapá, 1990.

12. Ministério da Saúde/Superintendência de Campanhas de Saúde Pública/DECEM/Divisão de Malária. Dados epidemiológicos da malária no Brasil de 1970 a 1989.

13. Neiva $A$. Uber die bildung einer chininresistent rase des malariaparasiten. Memórias do Instituto Oswaldo Cruz 2:131-140, 1910.

14. Payne D. Practical aspects of the use of the standard World Health Organization in vitro macro and microtest system for the determination of the sensitivity of Plasmodium falciparum to chloroquine, mefloquine, amodiaquine and quinine. MAP/ 84.2,01-17, 1984.

15. Payne D. Report on development of the World
Heath Organization standart microtest Kit for chloroquine, mefloquine, amodiaquine and quinine: 1979-1985. MAP/86.1,01-19, 1986.

16. Reys $\mathrm{S}$, Osanai $\mathrm{CH}$, Passos $\mathrm{AD}$. Resistência in vivo do Plasmodium falciparum as 4-aminoquinoleínas e a associação sulfadoxina + pirimetamina II. Estudos de Manaus, Amazonas 1983-1984. Revista Brasileira de Malariologia e Doenças Tropicais 38:37-44, 1986.

17. Rieckmann KH, Sax LJ, Campbel GH, Mrena JE. Drug sensitivity of Plasmodium falciparum. An in vitro microthecnique. The Lancet $1: 22-23,1978$.

18. Rodrigues DP. Casos de malária por Plasmodium falciparum resistentes ao tratamento pela cloroquina. Arquivos de Higiene e Saúde Pública 26:231-235, 1961.

19. Santos MAV, Couto AA, Oliveira SG, Rosário VE. Estudos in vitro dos níveis de resistência do Plasmodium falciparum a drogas, de 1983 a 1986. Revista do Instituto de Medicina Tropical de São Paulo 29:346-353, 1987.

20. Sawyer D. Frontier malaria in the Amazon region of Brazil. Type of malaria situation and some implication for control. Working paper prepared for the meeting of TDR/FIELDMAL/SC/AMAZON. 88(3): 1988.

21. Silva JR, Lopes PFA, Ferreira LFF, Morteo R, Naveira JB. Resitência do Plasmodium falciparum à ação da cloroquina. O Hospital 60:581-594, 1961.

22. Targett GAT. Chemotherapy and the immune response in parasitic. Parasitology 90:661-673, 1985.

23. Trager $W$. Jansen JB. Human malaria parasites in continous culture. Science 193:673-675, 1976.

24. Vasconcelos MA, Rosário VE. Testes de sensibilidade in vitro de amostras de Plasmodium falciparum da bacia Amazônica (Brasil). Revista Brasileira de Malariologia e Doenças Tropicais 35:21-28, 1983.

25. White NJ, Looareesuwan $S$, Warrel DA, Warrel MJ, Bunnag D, Harinasuta T. Quinine pharmacokinetics and toxity in cerebral and uncomplicated falciparum malaria. American Journal of Medicine 73:564-572, 1982.

26. World Health Organization Instructions for use the micro test kit for the assessement of the response of Plasmodium falciparum to chloroquine and mefloquine in vitro. MAP/82.1,1-9, 1982.

27. World Health Organization in vitro micro-test (mark II) for the assessement of the response of Plasmodium falciparum to chloroquine, mefloquine, quinine, sulfadoxine-pyrimethamine and amodiaquine. MAP/ 87.2,1-21, 1987.

28. Young MD, Moore DV. Chloronique resistance in Plasmodium falciparum. The American Journal of Tropical Medicine and Hygiene 317-320, 1961. 